\title{
Conceptualisations of infinity by primary pre-service teachers
}

\author{
Elizabeth Date-Huxtable ${ }^{1,2}$ (D) Michael Cavanagh ${ }^{3}$. \\ Carmel Coady ${ }^{1} \cdot$ Michael Easey $^{4}$
}

Received: 17 November 2017 / Revised: 10 March 2018 / Accepted: 9 April 2018 /

Published online: 1 May 2018

(C) Mathematics Education Research Group of Australasia, Inc. 2018

\begin{abstract}
As part of the Opening Real Science: Authentic Mathematics and Science Education for Australia project, an online mathematics learning module embedding conceptual thinking about infinity in science-based contexts, was designed and trialled with a cohort of 22 pre-service teachers during 1 week of intensive study. This research addressed the question: "How do pre-service teachers conceptualise infinity mathematically?" Participants argued the existence of infinity in a summative reflective task, using mathematical and empirical arguments that were coded according to five themes: definition, examples, application, philosophy and teaching; and 17 codes. Participants' reflections were differentiated as to whether infinity was referred to as an abstract (A) or a real (R) concept or whether both (B) codes were used. Principal component analysis of the reflections, using frequency of codings, revealed that $\mathrm{A}$ and $\mathrm{R}$ codes occurred at different frequencies in three groups of reflections. Distinct methods of argument were associated with each group of reflections: mathematical numerical examples and empirical measurement comparisons characterised arguments for infinity as an abstract concept, geometric and empirical dynamic examples and belief statements characterised arguments for infinity as a real concept and empirical measurement and mathematical examples and belief statements characterised arguments for infinity as both an abstract and a real concept. An implication of the results is that connections between mathematical and empirical applications of infinity may assist pre-service teachers to contrast finite with infinite models of the world.
\end{abstract}

Elizabeth Date-Huxtable

liz.datehuxtable@gmail.com

1 School of Computing, Engineering and Mathematics, Western Sydney University, Sydney, Australia

2 Cardiff, Australia

3 Department of Educational Studies, Macquarie University, Sydney, Australia

4 School of Education, Australian Catholic University, Brisbane, Australia 
Keywords Infinity - Mathematical conceptualisation - Conceptual change - Inquirybased learning

The proliferation of constructivist views of conceptual development has led to divergent conclusions about how mathematical concept construction and conceptual change occur (Dubinsky et al. 2005a, 2005b; Mitchelmore and White 2007; Pegg and Tall 2010; Scheiner and Pinto 2014). These views have recently led to development and evaluation of mathematics curricula in innovative contexts, which emphasise openended inquiry and reflection and which support the deep mathematical knowledge required for mathematics teaching in both school and teacher education programs (Cordeiro 1988; Harel and Sowder 2005; Iannone and Cockburn 2008; Kattou et al. 2010; Kolar and Čadež 2012; Singer and Voica 2003, 2008). Development of school curricula in Australia and elsewhere has highlighted the importance of stimulating conceptual mathematical thinking from the early school years (Cordeiro 1988; Harel and Sowder 2005; Iannone and Cockburn 2008). A conceptual approach has been shown to be successful when topics in algebra, geometry and calculus are introduced in secondary school and at university (Cavanagh 1996; Jirotková and Littler 2003, 2004; Pehkonen et al. 2006).

The study of infinity as a concept in mathematics has remained remote in formal school curricula (Monaghan 2001) and is under-represented in teacher professional development programs (Kattou et al. 2010; Yopp et al. 2011). Although potentially a powerful example for developing conceptual mathematical thinking, the concept of infinity is also readily misinterpreted and misrepresented, being strongly influenced by tacit models and teachers' and/or students' conflicting beliefs (Fischbein 2001; Iannone and Cockburn 2008; Yopp et al. 2011).

Attempts to unravel intuitive and learned understanding of infinity and its use in mathematics teaching span the last four decades (Fischbein 2001; Fischbein et al. 1979; Monaghan 1986, 2001; Tall 2001, 2007; Yopp et al. 2011). This research has revealed the prevalence of 'natural' and tacit models of infinity that result from intuition and pathways of concept construction that conflict with or challenge mathematics learning (Fischbein 2001; Fischbein et al. 1979). Despite this research activity, questions remain about mathematical concept construction and learning pathways for young people (i.e. pre-tertiary students, who are generally less than 19 years of age) that lead to unambiguous use of infinity in mathematical thinking; for example, clarity of algebraic understanding of infinity does not necessarily coincide with development of geometric comprehension (Kattou et al. 2010; Kolar and Čadež 2012; Monaghan 2001; Singer and Voica 2003, 2008; Yopp et al. 2011).

This study aims to fill the gap in the research literature about concept construction by investigating and describing concepts of infinity held by pre-service teachers, who were engaged in an online learning module, which was purposefully designed during development of an integrated science and mathematics program to stimulate inquiry, reflection and conceptual thinking about infinity (Mulligan et al. 2017; Mulligan et al. 2014). This paper addresses the question, "How do preservice teachers conceptualise infinity mathematically?" and reports an empirical investigation of how primary pre-service teachers conceptualise infinity after experiencing the module's learning activities. It examines the arguments and 
models that pre-service teachers use to portray numbers and theoretical objects of infinite and infinitesimal size, their arguments relating to measuring finite objects of very small or large size and their ability to identify the finite limits to measurement. Pre-service teachers' portrayals and arguments in the form of an extended response to a stimulus question, which were constructed under test conditions but based on a summary they prepared before the assessment, were interpreted as authentic expressions of concepts that were formed or developed during the learning activities in the module. The use of extended responses is regarded as a reliable method of evaluating the learning of complex ideas (Biggs and Collis 1982).

\section{Research on conceptualisations of infinity}

The conceptual development of infinity was thought to begin at about 14 years of age (Bruner 1966). More recently, it was considered to develop from about the age of 11-12 years, as observed in late primary and early secondary school students (Fischbein et al. 1979; Harel and Sowder 2005). However, other research has highlighted the diversity of performance on tasks related to infinity within all age groups during the school years (and later) and the complexity of factors contributing to this. Contributing factors include the influence of their teachers' beliefs about infinity, students' prior understanding of this complex concept (Monaghan 2001) and the dominance of procedural over conceptual learning (Tall 2007). Other studies indicate that when confronted by new and different concepts, individuals may return to earlier intuitive ways of thinking (Fischbein 2001; Tall 2007).

In relation to current theory of mathematical concept construction, the concept of infinity is theoretically entwined with almost every branch of mathematics (Tall 2001). Several research groups have examined students' quite disparate levels of understanding and application of infinity in different areas of mathematics (Jirotková and Littler 2003, 2004; Kattou et al. 2010; Kolar and Čadež 2012). In the following sections, the important role that these learning pathways play in relation to the formation of mathematical concepts, such as potential and actual infinity, types of infinity, static and dynamic modelling of infinite processes and extension of finite scales to the infinite, are introduced and in later sections discussed as to their use by pre-service teachers in this study.

Potential and actual infinity Young people's intuitions of infinity are usually of unbounded and never-ending processes, such as 'the numbers - or lines - go on forever'. The mental image formed is of an 'unfinishable' process or potential infinity. In contrast the mental image of actual infinity is an object, a complete entity. The realisation that there is no largest or smallest number or furthest or closest point in space in the abstract world is the catalyst to realising that actual infinity is not a number, though it has the number-like property of size. In this concept construction process, 'actions-first' and reflective abstraction appear to be dominant (Dubinsky et al. 2005b; Pegg and Tall 2010), because the young person progresses from understanding infinity as a process to 'encapsulating' the infinite process as an object. This growth in mathematical knowledge or ability is thought to be a sequential pathway. Each stage of the pathway forms the basis of a more advanced 'cycle' of learning, such that each 
concept learned is the object 'actioned' at the lowest level of the next, more sophisticated mathematical process (Dubinsky et al. 2005b; Pegg and Tall 2010).

Types of infinity The dichotomy of infinite and infinitesimal is of abstract as opposed to concrete size. Differences in size can also be distinguished between infinite sets of rational and irrational numbers using one-to-one correspondence methods, though these are unquantifiable in an absolute sense. Realisation that infinite properties can be qualitatively and even relatively different is more likely to employ 'objects first' and structural abstraction (Scheiner and Pinto 2014), because understanding one type of infinity (e.g. counting of whole numbers) does not necessarily lead sequentially to understanding another type (e.g. fractions between zero and one). Scheiner and Pinto (2014) proposed that each physical or mental object is acted upon in a variety of contexts via empirical or theoretical abstraction (Mitchelmore and White 2007) until the mathematical process becomes 'concrete', at which point the underlying structure of a concept is understood. By this process, 'types of infinity' are the 'mental objects' acted upon in tandem, and knowledge construction occurs by placing the newly acquired conceptual understanding within the learner's body of knowledge and restructuring them to 'make sense' of the combined knowledge (Scheiner 2016).

Static and dynamic models Inappropriate modelling of finite phenomena on an infinite scale can result in well-known paradoxes, such as the tale of 'the Hare and the Tortoise' in which the tortoise is given a large head start in a race between the two animals. During the time that the hare has moved half the distance to the tortoise, the tortoise has moved forward, and so on, such that the hare apparently never catches up to the tortoise. Confusion results because, although the spatial model of this paradoxical tale is infinite(simal), it is imposed on a temporal one, which is finite. In representing a dynamic problem mathematically, the student needs to use structural abstraction in clarifying which dimension is modelled on an infinite scale, for example, distance, and which one is finite, for example, time (Fischbein 2001; Monaghan 2001). It allows them to use different thought processes concurrently in juxtaposing the infinite and finite dimensions. As described above, these thought processes can occur in tandem via structural abstraction in which the newly acquired conceptual understanding of infinite models and more established understandings of the finite models of the world are restructured to make sense of the combined knowledge (Scheiner 2016).

Extension of the finite Human experience is finite and our mental models are strongly influenced by our experience. However, our experiences in the abstract world can be unrestricted by scale, such that we can imagine a large or small, but undefined, finite number or size, such as

- Counting - the number of biological niches or the number of grains of sand on Earth or the number of stars in the universe, all of which depend on defining the unit of counting

- Measuring - the size of the universe or a sub-atomic particle or photon of light, all of which depend on our ability to define boundaries that separate entities and to estimate within these boundaries accurately 
- Geometry - the space within three-dimensional shapes of objects that changes with the dimensions of time and gravity, such as in the presence of a 'Big Bang' or a 'black hole'

- Limits - that are relative to the scale we are working at, such as the volume of Earth's atmosphere and the proportions of gases contained when defining a 'liveable' state.

In such scenarios, both processes and objects are fluid in number and/or size in our mental models, but to work with them in the abstract, people represent a potential infinity with a finite entity. To work with actual infinite abstract entities, infiniteness must be recognised as an alternate state rather than 'more of the same'. As described in the previous sections, students make sense of differences between finite and infinite models of the world, when such thought processes can occur in tandem via structural abstraction (Scheiner 2016). However, this appears to be the main source of the difficulties students face in coming to terms with the concept of infinity (Monaghan 2001).

The present study focuses on a topic that is typically not addressed in a specific sense in primary pre-service teacher education programs - the concept of infinity. Whilst some previous studies have considered conceptions of infinity, none have addressed the issue of researching conceptions of infinity through real-life problems and situations in an integrated manner, drawing on science and mathematics applications, as the present study does. Hence, the results also provide some valuable implications for pre-service teacher programs.

\section{Research design and methodology}

This study reports one outcome from a trial of a learning module that is part of a large Australian government-funded project to develop integrated programs in science and mathematics for pre-service teachers, the Opening Real Science project (Mulligan et al. 2017; Mulligan et al. 2014). The module, called ' 2 Infinity and Beyond', was specifically designed to stimulate pre-service teachers to think conceptually about mathematics, by introducing it in scientific contexts that extend the limits of the real world, such as those in Table 1. The examples used in the module (indicated by an asterisk in Table 1) attempt to support the conceptualisation processes introduced in the previous section.

\section{Learning module design: 2 Infinity and Beyond}

A module of learning activities which integrates mathematical and scientific ideas in authentic contexts was designed to explore the concept of infinity, from its historical beginnings to some modern applications in science contexts (Table 1). The module was designed to be completed over a 4-week period within a unit of one university semester and delivered in online or blended mode. The resources embedded within the module were intended to stimulate discussion about infinity amongst pre-service teachers during their preparation for teaching, and it was trialled in blended learning mode with a class of fourth year primary pre-service teachers $(n=22)$. The module development team (MDT) consisted of a tertiary-level mathematician, a mathematics teacher educator and an educational developer. A Design Develop Implement (DDI) process was 
Table 1 Suggested science contexts for embedding the mathematics of infinity

\begin{tabular}{|c|c|c|}
\hline Form of infinity & Mathematical examples & Scientific examples \\
\hline $\begin{array}{l}\text { Numeric - rational vs. irrational } \\
\text { number sets/discrete vs. } \\
\text { continuous }\end{array}$ & Hilbert's hotel & Resource 'partitioning' \\
\hline $\begin{array}{l}\text { Geometric - extending finite } \\
\text { models }\end{array}$ & $\begin{array}{l}\text { Paper folding/cutting; Von Koch } \\
\text { snowflake/Sierpinski gasket } \\
\text { fractals }\end{array}$ & $\begin{array}{l}\text { Discovery of sub-atomic particles; } \\
\text { computer-generated imagery }\end{array}$ \\
\hline $\begin{array}{l}\text { Measurement-limits of } \\
\text { accuracy, approaching limits }\end{array}$ & $\begin{array}{l}\text { Circle circumference; coastline } \\
\text { paradox }\end{array}$ & $\begin{array}{l}\text { Wave-particle theory of light; } \\
\text { atmospheric } \mathrm{CO}_{2} \text { concentration* }\end{array}$ \\
\hline $\begin{array}{l}\text { Dynamic (infinite variation of } \\
\text { more than one factor) }\end{array}$ & $\begin{array}{l}\text { Zeno's paradoxes, e.g. the Hare } \\
\text { and the Tortoise race }\end{array}$ & $\begin{array}{l}\text { Universe-multiverse (black hole) } \\
\text { controversy*, Fermi paradox, } \\
\text { Drake equation }\end{array}$ \\
\hline
\end{tabular}

*Scientific examples used in the innovative integrated science and mathematics module of the Opening Real Science project (Mulligan et al. 2017; Mulligan et al. 2014)

implemented by completing design, development, trialling and enhancement phases of this process punctuated by a series of workshops to critically review the module's progress (Seeto and Vlachopoulos 2015). During the DDI process, the module was peer reviewed by mathematicians, mathematics teacher educators and educational developers and then revised. Revision focused on clarifying the online narrative to provide a clear rationale and progression through the topics, learning activities and formative assessments to the final summative assessment.

The MDT focused on both scoping and depth of content and the assessment of students' notions of infinity. They mapped to the module design:

- Content:

i. Identifying (mis)conceptions of infinity,

ii. Researching the history of infinity,

iii. Describing the types of infinity,

iv. Applying the concept of infinity in scientific contexts in which pre-service teachers examine mathematical and/or scientific examples of Fibonacci numbers, pi, the continued fraction for $e$, fractals, calculus concepts (rates of change, limits), sequences and series and infinitesimals, and

v. Reflecting on the usefulness of the concept of mathematical infinity in real-world contexts.

- Assessment:

i. Designing a short presentation (e.g. PowerPoint, video) investigating one misconception about infinity, discussing strategies to overcome the misconception in their teaching and

ii. Extending participants' application of conceptual mathematical thinking in scientific contexts in a longer summative assessment, which took the form of a critically reflective and evaluative discussion addressing the question, "Can anything real be infinite?", and in which module participants presented mathematical and empirical 
arguments for or against this question. These reflections provided data on participants' conceptualisations of infinity.

The inquiry-based 5Es model (engage, explore, explain, elaborate, evaluate) (Bybee 2009 , 2014) supported the development of the five topics. The module used the following teaching strategies: moderated face-to-face and online discussions to guide learning activities, preparation of a presentation and formative and summative reflective writing.

\section{Module learning activities}

Topic 1, ideas of infinity, contained stimulus material in videos and texts aimed at engaging the module participants in discussion and reflection in forums and blog posts. The forum and blog posts were used by the module tutor to diagnose any misconceptions and conflicting beliefs about the concept of infinity amongst the participants.

Topic 2, history of infinity, used discussion of stimulus material in podcasts and texts to support exploration of the mathematical concept of infinity. This topic culminated in a formative assessment in the form of a review quiz, which checked participants' recall and their conceptual understanding of historical and current ideas about mathematical infinity.

Topic 3, types of infinity, contained hands-on activities about numerical and geometric infinities, after which as a formative assessment, participants identified and discussed a misconception about infinity and explained how they would overcome it in a classroom or everyday setting.

Topic 4, applications of infinity, required module participants to elaborate on their learning through an inquiry activity about fractals and a modelling activity about carbon dioxide emissions, to explore and explain the differences between modelling of infinite and measurement of very large finite phenomena. This topic culminated in a second review quiz, which tested understanding of 'counting', 'geometric', 'measurement' and 'dynamic' applications of infinity in mathematical contexts (see Table 1).

Topic 5, reflection on infinity, was designed to elaborate participants' conceptual thinking about dynamic infinity in a specific scientific context, the origin of the universe (Table 1). They accessed a science blog (Mayes 2004) and were encouraged to use other material in the module, as well as undertaking their own research to collect and evaluate evidence in preparation for the summative assessment, a reflective discussion of the question, "Can anything real be infinite?"

\section{Trialling of the module with pre-service teachers}

Participants The module was trialled in blended learning mode with a class of fourth year primary pre-service teachers $(n=22)$, who were enrolled in a middle school mathematics unit within an Australian university's pre-service teacher education program. The unit was designed to develop and, in some cases, to enhance their mathematical knowledge. The class demographic included a broad range of ages and life experiences. Their tutor indicated that their levels of achievement in mathematics varied from completion of year 10 to university-level curricula. 
Procedures Participants accessed the module's resources online from the university's learning management system (LMS) for a week prior to interacting face-to-face in class. The latter delivery was an intensive week of full-time study, which included 1 day of face-to-face activities from the module and the summative assessment, that is, completion of the reflective discussion of the stimulus question, "Can anything real be infinite?" For their presentations in topic 3, instead of completing them individually, pre-service teachers formed groups of five to seven participants to investigate in-depth one of the activities in the module. They presented as a group to the rest of the class. Thus, the whole class experienced didactically all the activities their group did not investigate. During the preparation and presentation of their activities, participants were supported by their tutor, whose role was important in providing feedback and additional examples. Group presentations were audiovisually recorded and these were intended to be evaluated as participants' formative (or 'pre-test') conceptualisations of infinity. However, because some participants did not consent to use of this material, the study was determined to be descriptive and not experimental. With the assistance of the tutor and the module's educational developer, participants also completed the modelling activity in class. The activity was focused on developing an understanding of the usefulness of infinity in measurement accuracy in relation to predicting the limits to carbon dioxide emissions into Earth's atmosphere.

To complete the summative reflective task, participants were asked to prepare a hand-written summary of their research of topic 5 on one A4 sheet of paper. They could refer to the summary whilst completing their hand-written summative reflective task during the final $1 \mathrm{~h}$ session of the week of on-campus intensive study. They were also required to submit the summary with their reflection as a method of authenticating each individual's engagement in learning about the concept of mathematical infinity. Participants were told that there were no right or wrong answers to the stimulus question. They were guided by a rubric for the task that indicated that a high standard answer to the stimulus question would "draw on arguments raised in the stimulus article, other parts of the module and independently researched authoritative sources". It would also have "a well-argued, logical conclusion about the existence of infinity, demonstrating links between conceptual and numerical representations and applications of infinity".

Nineteen of the participants completed an online survey voluntarily and anonymously via a link at the end of the module upon completion of the module's activities. The online survey targeted the pre-service teachers' (PSTs') engagement in the module, not their conceptualisation processes. The engagement data collected, the tutor's evaluation of PSTs' engagement and their preparation for the reflective assessment were used to determine whether the module activities had a negative impact on their learning. Participants' responses were positive overall (Table 2), and their level of engagement was supported by the tutor's evaluative feedback. He commented that participants were "anxious at the outset because they thought the content would be well beyond them. But by day 5 of the course students were comfortable and engaged in good discussion about a complex topic." All 22 PSTs submitted summaries with their summative reflections that indicated that they were engaged in attempting to understand the applications of mathematical infinity in the real world. It was concluded that the module activities were unlikely to have had a negative impact on their learning. 
Table 2 Participants' responses to Likert-scale items about the module, where ' 6 ' is strongly agree, ' 5 ' agree, '4' somewhat agree, ' 3 ' somewhat disagree, '2' disagree and ' 1 ' strongly disagree

\begin{tabular}{|c|c|c|c|c|}
\hline Likert-scale items & Mean $^{\mathrm{a}}$ & $\begin{array}{l}\text { Standard } \\
\text { deviation }\end{array}$ & $\begin{array}{l}\text { Percent who } \\
\text { agreed }^{\text {b }}\end{array}$ & $\begin{array}{l}\text { Percent who } \\
\text { disagreed }^{c}\end{array}$ \\
\hline 1. I felt engaged as soon as we started the module & 4.26 & 1.59 & $78.9 \%$ & $21.1 \%$ \\
\hline $\begin{array}{l}\text { 2. The module included a variety of activities } \\
\text { which allowed me to explore the topic }\end{array}$ & 4.68 & 1.35 & $84.2 \%$ & $15.8 \%$ \\
\hline $\begin{array}{l}\text { 3. I can now offer an accurate explanation of the } \\
\text { mathematical concepts covered }\end{array}$ & 4.21 & 1.36 & $78.9 \%$ & $21.1 \%$ \\
\hline $\begin{array}{l}\text { 4. The assessment task/s allowed me to demonstrate my } \\
\text { understandings of the topics }\end{array}$ & 4.32 & 1.29 & $78.9 \%$ & $21.1 \%$ \\
\hline $\begin{array}{l}\text { 5. I have a better understanding of the mathematical } \\
\text { thinking process }\end{array}$ & 4.11 & 1.52 & $68.4 \%$ & $31.6 \%$ \\
\hline $\begin{array}{l}\text { 6. I feel more confident designing learning experiences } \\
\text { about mathematical concepts }\end{array}$ & 4.05 & 1.51 & $68.4 \%$ & $31.6 \%$ \\
\hline 7. Completing this module was relevant to my studies & 4.42 & 1.43 & $78.9 \%$ & $21.1 \%$ \\
\hline $\begin{array}{l}\text { 8. I would recommend completing this on-line module } \\
\text { to a fellow student }\end{array}$ & 4.26 & 1.59 & $73.7 \%$ & $26.3 \%$ \\
\hline
\end{tabular}

\footnotetext{
${ }^{\text {a }}$ A mean score of 4 and above (out of 6) on each item is set as the benchmark to indicate that students are happy with the module; a mean score of less than 3 indicates, on average, a negative response from students to the module and suggests that there are issues that need to be examined

${ }^{\mathrm{b}}$ Agree combines responses strongly agree, agree and somewhat agree

${ }^{\mathrm{c}}$ Disagree combines responses strongly disagree, disagree and somewhat disagree
}

\section{Qualitative analyses of responses to the summative reflective task: "Can anything real be infinite?"}

Module participants' demonstrations of their conceptualisation of infinity in their summative reflective tasks were categorised using an open approach, which was based on the coding of phrases used in their reflections and the grouping of these into themes.

Coding of phrases Codes were developed by comparing phrases of four randomly selected reflections, in consultation with the educational developer, the mathematics teacher educator and the tutor of the module trial. To test the validity and reliability of the coding scheme, the mathematics teacher educator and educational developer independently coded a fifth randomly selected reflection and then compared their codings. This process was repeated with three more randomly selected reflections. When differences in coding occurred, discussion ensued. In the process, refinements were made to the coding scheme, such that some codes were removed or collapsed together until agreement on a code and its definition was reached.

The following changes to codes were made during the inter-rater reliability process and examples are provided to illustrate these changes: 'application of infinity' was replaced by and separated into four 'empirical' examples. Samples of these are shown in the Appendix in the section (b) Examples. 'Numbers [are] abstract' was included in the 'abstract concept' code. A sample of the 'numbers are abstract' recoded to 'abstract 
concept' is “... numbers can go on forever, but numbers are not real, they are abstract" (participant 6). 'Extension of finite' to infinite modelling or reasoning was included in comparisons of 'potential vs. actual' infinity. This last code was separated into 'potential infinity' and 'actual infinity', and these were then included in the 'without limit' and 'never reached' codes, respectively. An example of a statement that was at first classified as 'extension of finite' then 'potential vs. actual' then 'actual infinity' then 'never reached' is "... each time we delve into the concept we think we are getting closer to it, only to find we haven't reached it" and the example used to illustrate this, which was coded as 'empirical: geometric' was "One example of how fractals and infinity work together is when looking at a coastline on a map. Each time the magnification is increased more of the coastline is shown..." (participant 13). An example of a statement that was at first classified as 'extension of finite' then 'potential vs. actual' then 'potential infinity' then 'without limit' is "The existence of days, months, seasons and time in general. All of these concepts still are existing and are limitless and go on and on. We cannot see a finite end to these ideas" (participant 17). During the four successive co-coding sessions of reflections, agreement between the mathematics teacher educator and educational developer improved from 50 through 67 and 75 to $95 \%$ of their codings such that all further coding was undertaken solely by the educational developer.

The final set of 17 codes, which were grouped into 5 themes: definition, examples, application, philosophy and teaching, was defined with examples from module participants' summative reflective tasks in the Appendix. The educational developer (re)coded all 22 reflections according to this set of codes.

\section{Quantitative analysis: Trends in conceptualisation of infinity}

Each reflection was coded using the codings in the Appendix. The frequency of each code for each reflection was recorded in a spreadsheet. The frequency data for codings were normally distributed (Pearson $\chi_{(16)}^{2}=22.74, p=0.121$ ) and the variance was homogeneous (Bartlett $\left.\chi_{(136)}^{2}=175.91, p=0.012\right)$. The variables were not scaled before analysis, as they were on the same scale. These frequencies were then submitted to exploratory factor analysis, using the statistical program $\mathrm{R}$ to perform a principal component analysis. Principal component analysis with no rotation was undertaken to identify correlations amongst the codings of participants' summative reflections that described common patterns in demonstrations of the understanding of infinity by module participants. Loadings of variables (i.e. codes) on the principal components were used to determine which codes were most influential in differentiating amongst the summative reflections.

\section{Results}

\section{Frequency of coding of summative reflective tasks}

From the coding of 22 summative reflective tasks, a total of 437 codings was distributed across the reflections with the mean being approximately 20 codings for 
each reflection $($ mean $=19.86$, standard deviation $=4.89)$ and range 12 to 34 . The mean number of times that a code was used was approximately 26 (mean $=25.71$, standard deviation $=11.93$ ) with the range 10 to 43 . The frequencies and percentages of code use and the mean and standard deviation of code use per summative reflection are shown in Table 3. Higher percentages of codings of 'abstract concept', 'without limit' and 'never reached' than of 'real concept' codes (AC, WL, NR, RC) occurred amongst the reflections. 'Mathematical numerical' (M:n) examples were the most frequently used examples, other examples being less frequently used and involving longer, more complex explanations, particularly 'rules of arithmetic' and 'empirical dynamic' examples (RA, E:d). 'Theoretical vs. practical' (TvP) aspects of using infinity, that is, comparing its use in modelling vs. its application is the real world, were frequently used and approximately one quarter of the time 'technological limitations' (TL) of applying models of infinity in the real world were cited specifically. Many of the participants concluded with 'philosophical reflections' (PR), which included belief statements about whether infinity can be thought of as part of the 'real world' or not. Some also referenced 'social, cultural and religious' (SCR) beliefs or practices, mostly as an alternative non-mathematical, non-empirical interpretation of infinity. Some participants mentioned 'learning and teaching applications' of infinity (code LTA; Table 3).

\section{Trends in conceptualisation of infinity}

The results of the principal component analysis revealed two principal components (PC), which had eigenvalues greater than 4 and which explained $39.6 \%$ of the variation in the coding (PC1 21.8\%, PC2 17.8\%). The two principal components were used to differentiate the summative reflections according their coding patterns (Fig. 1). 'Abstract concept' loaded strongly positively and 'real concept' strongly negatively on principal component 1 (PC1; Table 4 and Fig. 1). Thus, a pattern emerged from this analysis, which was centred on the participants' argument that infinity is either an abstract or a real concept, or a combination of both. Three groups were identified such that their entire reflections were then labelled with a generic code: ' $A$ ', those coded only with 'abstract concept' $(N=9)$; ' $\mathrm{R}$ ', those coded only with 'real concept' $(N=7)$; or 'B' (both), those coded with both 'abstract concept' and 'real concept' codes $(N=6)$. Each reflection identified by its generic code was plotted in a biplot by its position on the two principal components (Fig. 1). The abstract generic code (A) was centred at the positive end of PC1 and the real generic code (R) was centred at the negative end, whilst summative reflections with both codes (B) were dispersed across the plot area (Fig. 1).

The strength and direction of loading of codes on the two principal components (Table 4) was shown by the direction and length of the arrows in Fig. 1. The 'Theoretical vs. practical' code was strongly positively correlated with principal component 1 . The 'without limit' code was moderately positively and the 'never reached', 'theoretical vs. practical' codes and 'empirical geometric' examples were moderately negatively correlated with principal component 2 (PC2; Table 4 and Fig. 1). These codes are used in the following section to describe the types of evidence and arguments correlated with each generic code (A, R or B). Where the correlation between a code and one or both principal components cuts across a group of points, indicated by either the A or R generic code, that code was used frequently in that group of reflections. For example, the group of reflective tasks that were coded with 'abstract concept' but not 'real concept' 


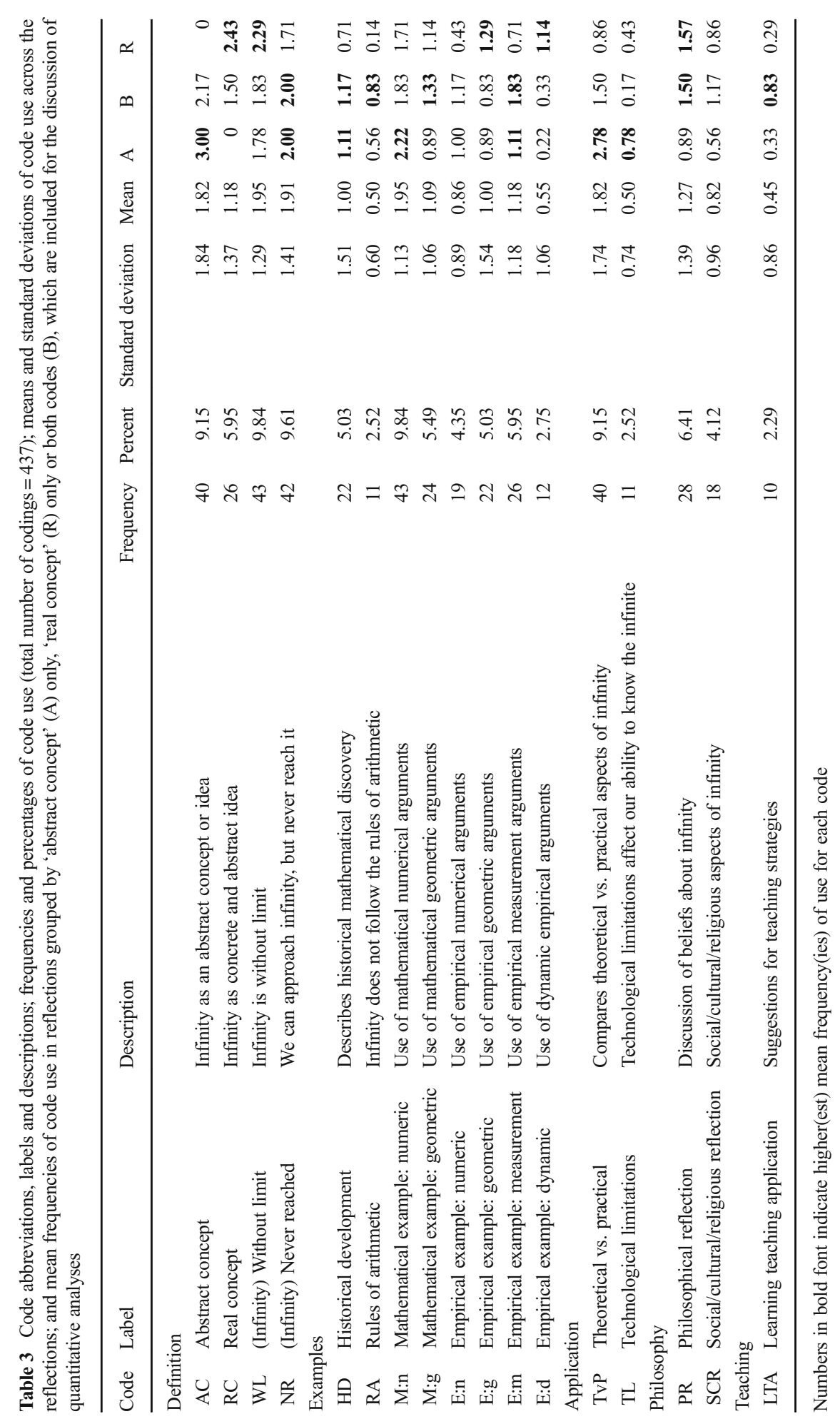


(generic code A) were very likely to be coded with 'theoretical vs. practical'. Similarly, the group of reflective tasks coded with 'real concept' but not with 'abstract concept' (generic code R) were very likely not to be coded with 'theoretical vs. practical'. Reflective tasks of the B generic code were likely to be coded with 'never reached', 'empirical geometric' and 'theoretical vs. practical' but not with the 'without limit' code.

\section{Qualitative arguments about infinity}

The participants used three distinct styles of argument and evidence: (i) mathematical and empirical applications and comparisons characterised arguments for infinity as an abstract concept, (ii) mathematical and empirical geometric and empirical dynamic examples and belief statements characterised arguments for infinity as a real concept and (iii) empirical measurement and mathematical examples and belief statements characterised arguments for infinity as both an abstract and a real concept.

Arguments for infinity as an abstract concept Amongst the reflections which stated that infinity is an abstract concept, for example "Infinity is a qualitative term used to describe the unmeasurable" (AC, participant 5), mathematical numerical examples were often used, for example "Bhaskara was a 12th century Indian mathematician who gave sense to the concept of infinity... as we divide by a smaller amount each time we are producing more and more pieces... if we could divide by zero, an infinite number of pieces would result" (M:n, participant 5). These reflections also tended to use empirical measurement examples, such as "the example used to demonstrate this philosophy, is that of a black hole, specifically the notion of it having infinite mass" (E:m, participant 15), and statements that referred to infinity as never reached: "we move closer towards our limits, however we don't come closer to infinity" (NR, participant 5). They also tended to draw on comparisons of theoretical and practical uses of infinity (TvP) and were more likely to make reference to practical and specifically technological limits (TL) that prevent more accurate measurement and a 'closer approach to infinity' (Fig. 1). For example,

"Our understanding of the world in which we live changes with advancement in technology. The realisation that all matter is made up of particles came with the invention of the microscope and these particles became known as atoms. As our ability to measure more accurately developed we could establish that these atoms were comprised of subatomic particles. As we move towards the perceived limits of measurability, our understanding and ability to make sense of things changes" (TL, participant 5).

And

"Mathematically speaking the mass of a black hole is represented by the expression of infinity. While this is the accepted measurement in contemporary science, it is out of the necessity to apply a quantity to that which cannot be quantified by current understanding of the universe. While a black hole is a tangible construct 


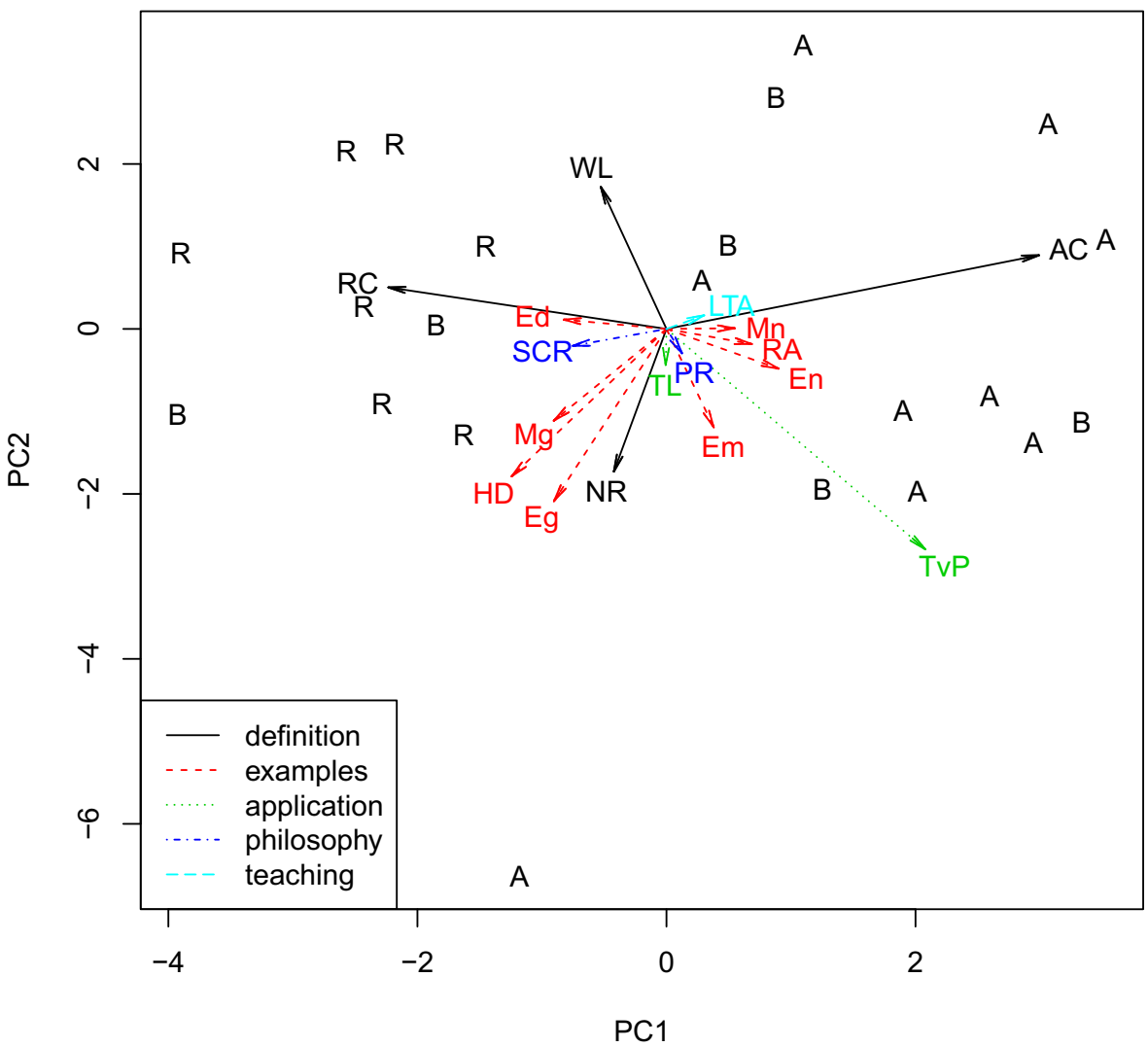

Fig. 1 Biplot, showing the loadings of the 17 variables (codes) on the first two principal components as arrows. Codes are indicated by their two- or three-letter abbreviations, and the legend indicates a code's theme (see Table 3). Each summative reflection is represented by its generic category: abstract (A), real (R) or both (B) concept codes, respectively. The Abstract category is centred at the positive end of PC1 and the Real category is centred at the negative end, whilst summative reflections with both codes are dispersed across the plot area

in theory, humans do not possess the technology to adequately survey and analyse such a phenomenon..." (TL, participant 15)

Pre-service teachers in this study who referred to infinity as an abstract concept were more likely to use arguments based on evidence of measurement of very large or very small, finite phenomena and contrasting the practical or technological limits of finite, measurable objects with theoretically infinite, immeasurable models than those who referred to infinity as a real concept.

Arguments for infinity as a real concept For reflections that referred to infinity as a real concept, for example "Based on the knowledge of infinity, its existence as having the capability to be real is highly possible" (RC, participant 22), empirical geometric and empirical dynamic examples were often used (Fig. 1). The pre-service teachers 
Table 4 Loadings of variables (codes) on the first two principal components

\begin{tabular}{llcr}
\hline Code & Label & Component 1 & Component 2 \\
\hline AC & Abstract concept & $\mathbf{0 . 6 0}$ & 0.18 \\
RC & Real concept & $-\mathbf{0 . 4 5}$ & 0.10 \\
WL & (Infinity) Without limit & -0.11 & $\mathbf{0 . 3 4}$ \\
NR & (Infinity) Never reached & -0.08 & $-\mathbf{0 . 3 5}$ \\
HD & Historical development & -0.25 & $\mathbf{0 . 3 6}$ \\
RA & Rules of arithmetic & 0.14 & -0.04 \\
M:n & Mathematcial example: numeric & 0.11 & 0.002 \\
M:g & Mathematical example: geometric & -0.18 & -0.22 \\
E:n & Empirical example: numeric & 0.18 & -0.10 \\
E:g & Empirical example: geometric & -0.18 & $-\mathbf{0 . 4 2}$ \\
E:m & Empirical example: measurement & 0.07 & -0.24 \\
E:d & Empirical example: dynamic & -0.16 & 0.02 \\
TvP & Theoretical vs. practical & $\mathbf{0 . 4 1}$ & $-\mathbf{0 . 5 3}$ \\
TL & Technological limitations & -0.001 & -0.09 \\
PR & Philosophical reflection & 0.02 & -0.06 \\
SCR & Social/cultural/religious reflection & -0.15 & -0.04 \\
LTA & Learning and teaching application & 0.06 & 0.03 \\
\hline & & & \\
\hline
\end{tabular}

Higher loadings are shown in bold font. Bold font also indicates 'Abstract concept' and 'Real concept' codes

who thought of infinity as a real concept were those who used complex empirical, real world examples. For example,

"The universe could not exist without time and time could not exist without the universe. ... If we move away from thinking of space and our universe as being a geometric sphere and just think of it as a multidimensional, unbounded area then you could imagine how it would be infinite" (E:d, participant 21).

And

"The technology we use now, uses scale to identify distance, however if we consider that 'large numbers are no nearer to infinity that small numbers' then we can instead focus on the infinite number of measurement possibilities. In class we were presented with the coastline paradox. Even though we could magnify the scales, we discovered that by using smaller measurements it would be closer to accurate.... There are options that haven't been thought of or we don't have access to yet which all contribute to the infinite possibilities to utilise as measurements" (E:g, participant 22).

By arguing with complex empirical examples, extending finite models to the infinite and concluding with belief statements, for example "If time is infinite then that leads me to believe that the universe is also infinite" (PR, participant 21), they differed from the pre-service teachers who referred to infinity as an abstract concept, and who 
frequently used mathematical and empirical measurement examples and comparisons but rarely used belief statements.

Arguments for infinity as abstract and real Reflections that referred to infinity as both abstract and real were not strongly associated with any codes, though they were moderately associated with 'never reached', 'empirical geometric' and 'theoretical vs. practical' (Fig. 1). The pre-service teachers who identified infinity as both abstract and real as a concept were possibly still developing their ideas of infinity, for example "... this has resulted in my view being that infinity is both a real and an unreal concept" (RC, AC, participant 8) and "I believe that something can be infinite but we have no way of proving it is" (RC, participant 14), and in relation to pi and the circumference of a circle "Both of these are valid but not real due to them both being mathematical (numbers)" (AC, participant 14). Alternatively, they may be deliberately 'keeping an open mind' and not excluding the possibility that proof of infinity may occur, which they considered currently beyond the explanatory power of mathematics and science, for example,

"Numbers can be seen as big or as small, but infinity isn't a number. No matter how big the biggest number you can think of [is], it is no closer to infinity than the smallest number. Infinity is a concept with no specific 'end', giving the term of a big number nothing compared to infinity ... In mathematics, infinity could be real. In the universe I still have an open mind to the fact it might be real. But we have no way of proving this" (M:n, participant 14).

In addition, the module participants who presented 'mixed messages' about infinity were those most likely to suggest applications of infinity for the school classroom. For example,

"It is important to show students in relation to infinity that depending on context, for example numbers and limits, that infinity no matter how hard we try we are able to become close but never reach it exactly. Georg Cantor's continuum hypothesis plays an important role in students' understanding of rational and irrational numbers. However, it isn't proven true or false which causes a conflict. I believe infinity is an abstract concept, when used in the appropriate context. This idea of infinity is yet to be proven, however it gives us the boundaries of mathematics" (M:n, LTA, participant 8).

These last two excerpts use mathematical numerical examples to demonstrate scientific reasoning, which acknowledges that bodies of knowledge can have different levels of certainty. Other pre-service teachers who referred to infinity as both abstract and real used empirical numerical, geometric and measurement examples, similarly to the pre-service teachers who referred to infinity only as an abstract concept. They did not use complex real-world examples involving the dimensions of both time and space, which the pre-service teachers who referred to infinity only as a real concept used. Unlike the previous two groups, these pre-service teachers demonstrated their thinking about introducing their students to theorising using mathematical infinity for modelling and predicting. 


\section{Discussion}

Pre-service teachers in this study who referred to infinity as an abstract concept contrasted finite models of the known world with the infinite as representing the immeasurable. This demonstrated development of the use of actual infinity to represent a state that is separate or alternative to the known (finite) world (Fischbein 2001). They demonstrated reflective abstraction when extending finite models to the infinite and then structural abstraction (Scheiner 2016) to make sense of the distinction between the finite world, which has been discovered through measurement (and technology), and the infinite, which is immeasurable. Pre-service teachers who thought of infinity as a real concept extended finite models of thinking to include the infinite (Fischbein 2001). They did not contrast finite, measurable objects with infinite, immeasurable phenomena, so that in making sense of this they sometimes concluded their evidence with belief statements about the existence of infinity. Similarly to pre-service teachers who thought of infinity as an abstract concept, they used reflective abstraction to extend finite models to the infinite; however, they made sense of this using structural abstraction (Scheiner 2016) in a different way by stating their belief that infinity exists. The group of teachers who referred to infinity as both an abstract and a real concept appeared to be using abstraction differently from the other two groups, who regarded infinity only as an abstract concept or a real concept. The use of a variety of numerical, geometric and measurement examples as evidence for their arguments suggests that they were using reflective abstraction by extending finite models to the infinite, but the use of belief statements as evidence led to an impression that their ideas about infinity were still forming and that they were in the process of making sense of their ideas via structural abstraction (Scheiner 2016). This last group of summative reflections demonstrated how beliefs that conflict with the mathematical idea of infinity can change its conceptualisation. Through examining the abstraction processes used by individual preservice teachers to conceptualise mathematical infinity, this study has provided new insights into the way reflective and structural abstraction (Scheiner 2016) can shape understanding of this important mathematical concept.

\section{Limitations}

From the quantitative and qualitative analyses, it is evident that individuals choose different types of mathematical and empirical examples at different frequencies to support arguments about the existence of infinity. For example, frequencies of use of the 'without limit' and 'never reached' codes were different amongst the groups of reflections and supported different conclusions.

It has been documented previously that the context in which infinity is introduced (in the classroom) has a strong influence on its mathematical conceptualisation (Tall and Tirosh 2001). In this study, such contexts, numerical, geometric, measurement and dynamic (Monaghan 2001), were introduced non-didactically to provide participants with opportunities to articulate their own ideas and to formulate their own arguments. These opportunities were provided during forum posts and informal class discussions (Harel and Sowder 2005), during group presentations to the class (Mitchelmore and White 2007) and during individual research for the summative reflective task, all of which are thought to be consistent with encouraging reflective and structural abstraction (Scheiner 2016). 
Participants may also have been influenced by previous experiences and intuitions in relation to infinity (Fischbein 2001; Tall 2001) and any independent research they did during the module, which was encouraged. As mentioned previously, an opportunity to identify participants' intuitions and (mis)conceptions about infinity was available during the first discussion forum in the module. However, a quiz or survey that targets diagnosis of misconceptions in relation to specific mathematical and empirical examples (see Table 1 and Appendix) would be a valuable addition to the beginning of the module as a baseline to compare with use of examples and their interpretations in the summative reflective task. This would then facilitate the documenting of any conceptual change that occurs during the module (Lane 2015).

It has been suggested that to regard infinity as a process is a more intuitive way of thinking than regarding it as an object or state (Fischbein 2001; Tall 2001). This was reflected in the greater use of 'without limit' and lesser use of 'never reached' codes in the reflections that regard infinity as a real concept compared with those that regard infinity as an abstract concept. Interestingly, those reflections which considered infinity as both a real and an abstract concept used the 'never reached' more than the 'without limit' code. However, this last group of summative reflections was more likely to revert to belief statements rather than to conclude with a summary of the evidence in their philosophical reflections.

Participants, who referred to the concept of infinity as both an abstract and a real concept, were also more likely to be inconclusive about the nature of infinity and they cited the influence of context (see participant 8's quote above). To ensure that module participants can explain their understanding of infinity in different mathematical and scientific contexts, the review quiz at the end of topic 4 could be revised to more accurately and comprehensively target specific numerical, geometric, measurement and dynamic contexts (Monaghan 2001), in order to prepare students for evaluating these sources of evidence in their reflections. This could then facilitate formative assessment of individual participants' progress via comparison with the (proposed) diagnostic quiz at the beginning of the module (Lane 2015).

\section{Implications and conclusion}

An implication of the results of this study is that connections between mathematical and empirical (scientific) examples of the applications of infinity, particularly numerical and geometric ones, may assist pre-service teachers to contrast finite with infinite models of the world. That is, the module activities may assist some of the module participants to regard infinity as an abstract entity, by which unknown quantities and qualities can be represented. After the module is revised to incorporate diagnostic and formative (in addition to review) quizzes and to add examples that explicitly connect mathematical and scientific applications of infinity, more participants should be able to demonstrate these thought processes, potentially extending their philosophical reflections to 'reflucturally' abstract levels (Scheiner 2016). Further testing of the module's support of such higherorder thinking processes in a larger cohort of pre-service teachers, using the detailed understanding gained from this study of participants' responses to the module's learning activities, will form the basis of future investigations into its pedagogical structure. 
The diverse contexts provided by modern scientific investigations of the 'very large' and 'very small', which were used in this study (e.g. Table 1), may be desirable settings for philosophical reflection and debate about the concept of mathematical infinity. Such debate about infinity, when encouraged in the mathematics classroom, could in turn support the position of mathematics within the science-technology-engineeringmathematics (STEM) curriculum as an important tool for driving the development of design thinking and innovation (Office of the Chief Scientist 2013).

Acknowledgements Support for this project has been provided by the Australian Government Department of Education and Training for Opening Real Science: Authentic Mathematics and Science Education for Australia (ORS), part of Enhancing the Training of Mathematics and Science Teachers (ETMST) (20132017). The views expressed in this publication do not necessarily reflect the views of the Australian Government Department of Education and Training. This paper was improved by the pedagogical advice of Katherine Stewart, statistical advice of Russell Thompson, and editorial advice of Leanne Rylands, Joanne Mulligan, and anonymous reviewers.

\section{Appendix}

Codes used to document five themes: (a) definition, (b) examples, (c) application, (d) philosophy and (e) teaching in primary pre-service teachers' summative reflections arguing an evidence-based answer to the question, 'Can anything real be infinite?', showing abbreviations, labels, descriptions and examples

Code Label Description Pre-service teachers' examples

(a) Definition

\section{AC Abstract concept}

RC Real concept

WL (Infinity) Without limit

NR (Infinity) Never reached We can approach (get closer and closer to) the infinite, but never reach it/prove its existence in the real world

(b) Examples

HD Historical development
Infinity as an abstract concept or idea

$$
\begin{aligned}
& \text { Infinity is an abstract concept ... } \\
& \text { Infinity ... is not a real number. It is } \\
& \text { an idea. }
\end{aligned}
$$

Infinity as real concept is inclusive of I believe that infinity is not a real concrete and abstract ideas

Infinity is without limit/limitless/unbounded and hence cannot be measured number, however it is real concept

Infinity is without a limit or boundary; it cannot be measured or counted. For example, the sequence of whole numbers beginning from zero is infinite because there is no whole number that is the greatest. It is a concept of limitlessness; it is not an amount.

The line gets closer and closer to the axes but never touches them.

...you could approach infinity, but never reach it. In this case, we would get very close to the wall but not touch it.

We must consider the history of number...

Modhava was a 15 th century Indian mathematician whose work unlocked the precise nature of pi. 
(continued)

Code Label Description $\quad$ Pre-service teachers' examples

RA Rules of arithmetic

M:n Mathematical example: numeric

M:g Mathematical example: geometric

E:n Empirical example: numeric

E:g Empirical example: geometric

E:m Empirical example: measurement

\section{E:d Empirical example: dynamic}

(c) Application

TvP Theoretical vs. practical Theoretical vs. practical aspects of infinity

Use of empirical arguments (i.e. maths embedded in science): measurement dynamics of more than one dimension
Infinity does not follow the arithmetical rules ... therefore you cannot include as a normal number and expect the rules of arithmetic to hold.

...when trying to match [using 1:1 correspondence] decimals with whole numbers you are unable to list them all due to there being an infinite number of possibilities.

Geogebra was used to construct polygons in the inside and outside of a circle... The relationship between the perimeters of the shapes and the diameter of a circle approached pi as the number of sides increased.

...how blood cells propagate themselves...scientists investigated the infiniteness of blood cells appearing in the real world with maths $\left(2^{\mathrm{n}}\right)$.

One example of fractals and infinity working together is when looking at a coast line...each time the magnification is increased more of the coastline is shown.

...a black hole is a star that has condensed, meaning that it has density but too high for us to measure

Before Einstein people thought that the speed of light was infinite, but then Einstein explained that the speed was equal to $E=\mathrm{mc}^{2} \ldots$ being finite.

Zeno's paradox of the race between Achilles (or a hare) and a tortoise...

Whilst we live in finite reality, we do accept that there are things that can theoretically be infinite ...

The problem is that you cannot get into a black hole and measure it. You would die and the world would end before you finished measuring it ...

However, often once answers are found, what we once thought was infinite, becomes finite. 
(continued)

\begin{tabular}{|c|c|c|c|}
\hline Code & Label & Description & Pre-service teachers' examples \\
\hline TL & $\begin{array}{c}\text { Technological } \\
\text { limitations }\end{array}$ & $\begin{array}{l}\text { Technological limitations affect our } \\
\text { ability to know the infinite }\end{array}$ & $\begin{array}{l}\text { We may reduce/discover an infinite } \\
\text { "item" but we are bound by hu- } \\
\text { man limits. Our own ability in } \\
\text { technological development and } \\
\text { advancements limit us in truly } \\
\text { discovering our own limits. }\end{array}$ \\
\hline
\end{tabular}

(d) Philosophy

PR Philosophical reflection $\begin{gathered}\text { Philosophical reflection: discussion } \\ \text { of beliefs (vs. evidence only) in } \\ \text { relation to infinity and } \\ \text { mathematics }\end{gathered}$

There is strong evidence...that supports the belief in the concept of infinity and that it does exist in a theoretical or conceptual form.

After numerous studies we have not mastered infinity... what I believe is that infinity is a concept we need to put things in perspective.

I believe we are so close to understanding infinity; however, we will never truly understand, so close yet so far. It's a term to show what we do not know or have not explored.

SCR Social/cultural/religious
reflection $\quad \begin{gathered}\text { Social/cultural/religious aspects of } \\ \text { infinity }\end{gathered}$

(e) Teaching
LTA Learning and teaching application

Learning and teaching application: consideration/suggestions for teaching strategies
For me as a Catholic and life after death, forever in heaven means exactly that; no limit, endlessness, boundlessness.

Sometimes it comes done to faith!

In linking this to the classroom... through elements of discovery learning we can help students grasp the concept of infinity.

It is a good way to show students by manipulating materials to understand the concept and to discuss the relationship between infinity, science and mathematics.

\section{References}

Biggs, J., \& Collis, K. (1982). Evaluating the quality of learning: the SOLO taxonomy. New York: Academic Press.

Bruner, J. (1966). Toward a theory of instruction. Cambridge: Harvard University Press.

Bybee, R. W. (2009). The BSCS 5E instructional model and 21st century skills (Paper commissioned for the Workshop on Exploring the Intersection of Science Education and the Development of 21st Century Skills.). Washington, D.C: National Academies Board on Science Education.

Bybee, R. W. (2014). NGSS and the next generation of science teachers. Journal of Science Teacher Education, 25, 211-221. https://doi.org/10.1007/s10972-014-9381-4.

Cavanagh, M. (1996). Student understandings in differential calculus. Paper presented at the Technology in mathematics education, Melbourne, Australia, 30 June-3 July, 1996.

Cordeiro, P. (1988). Playing with infinity in the sixth grade. Language Arts, 65(6), 557-566. 
Dubinsky, E., Weller, K., McDonald, M., \& Brown, A. (2005a). Some historical issues and paradoxes regarding the concept of infinity: an APOS-based analysis: Part 1. Educational Studies in Mathematics, 58, 335-359. https://doi.org/10.1007/s10649-005-2531-z.

Dubinsky, E., Weller, K., McDonald, M., \& Brown, A. (2005b). Some historical issues and paradoxes regarding the concept of infinity: an APOS-based analysis: part 2. Educational Studies in Mathematics, 60, 253-266. https://doi.org/10.1007/s10649-005-2531-z.

Fischbein, E. (2001). Tacit models and infinity. Educational Studies in Mathematics, 48, 309-329.

Fischbein, E., Tirosh, D., \& Hess, P. (1979). The intuition of infinity. Educational Studies in Mathematics, 10, 3-40.

Harel, G., \& Sowder, L. (2005). Advanced mathematical-thinking at any age: its nature and its development. Mathematical Thinking and Learning, 7(1), 27-50.

Iannone, P., \& Cockburn, A. D. (2008). "If you can count to ten you can count to infinity really": fostering conceptual mathematical thinking in the first year of primary school. Research in Mathematics Education, 10(1), 37-51.

Jirotková, D., \& Littler, G. (2003). Student's concept of infinity in the context of a simple geometrical construct. Paper presented at the proceedings of the 27 th conference of the International Group for the Psychology of mathematics education, University of Hawaii, Honolulu.

Jirotková, D., \& Littler, G. (2004). Insight into pupils' understanding of infinity in a geometrical context. Paper presented at the proceedings of the 28th conference of the International Group for the Psychology of mathematics education, Bergen, Norway, 14-18 July, 2004.

Kattou, M., Thanasia, M., Kontoyianni, K., Constantinos, C., \& George, P. (2010). Teachers' perceptions about infinity: a process or an object? Paper presented at the sixth congress of the European Society for Research in mathematics education, Lyon, France, 28 January-1 February, 2009.

Kolar, V. M., \& Čadež, T. H. (2012). Analysis of factors influencing the understanding of the concept of infinity. Educational Studies in Mathematics, 80, 389-412. https://doi.org/10.1007/s10649-011-9357-7.

Lane, R. (2015). Primary geography in Australia: pre-service primary teachers' understandings of weather and climate. Review of International Geographical Education Online, 5(2), 199-217.

Mayes, K. (2004). Theories with problems. Retrieved from http://www.thekeyboard.org.uk/index.htm.

Mitchelmore, M., \& White, P. (2007). Abstraction in mathematics learning. Mathematics Education Research Journal, 19(2), 1-9.

Monaghan, J. (1986). Adolescents' understanding of limits and infinity. Coventry: PhD Thesis, The University of Warwick.

Monaghan, J. (2001). Young people's ideas of infinity. Educational Studies in Mathematics, 48, $239-257$.

Mulligan, J., Cavanagh, M., Geiger, V., Hedberg, J., Pask, H., Rylands, L., et al. (2017). Opening real science: authentic mathematics and science education for Australia. Final report. Canberra, ACT: Australian government Department of Education and Training.

Mulligan, J., Hedberg, J., Parker, Q., Coady, C., \& Cavanagh, M. (2014). Opening real science: introducing authentic scientific methodology into mathematics and science teacher preparation. Paper presented at the science technology engineering and mathematics conference: Education and our planet: Making connections across contexts, University of British Columbia, Vancouver.

Office of the Chief Scientist. (2013). Science, technology, engineering and mathematics in the national interest: a strategic approach. Canberra: Australian Government.

Pegg, J., \& Tall, D. (2010). The fundamental cycle of concept construction underlying various theoretical frameworks. In L. E. B. Sriraman (Ed.), Theories of mathematics education: advances in mathematics education (pp. 173-192). Berlin: Springer-Verlag.

Pehkonen, E., Hannula, M. S., Maijala, H., \& Soro, R. (2006). Infinity of numbers: how students understand it. Prague: Paper presented at the International Group for the Psychology of Mathematics Education.

Scheiner, T. (2016). New light on old horizon: constructing mathematical concepts, underlying abstraction processes, and sense making strategies. Educational Studies in Mathematics, 91, 165-183. https://doi. org/10.1007/s10649-015-9665-4.

Scheiner, T., \& Pinto, M. M. F. (2014). Cognitive processes underlying mathematical concept construction: the missing process of structural abstraction. Paper presented at the joint meeting of PME 38 and PMENA 36, Vancouver, Canada,

Seeto, D., \& Vlachopoulos, P. (2015). Design Develop Implement (DDI)—a team-based approach to learning design. Paper presented at the The Higher Education Technology Agenda, Gold Coast, Australia, 11-13 May 2015.

Singer, F. M., \& Voica, C. (2003). Perception of infinity: does it really help in problem solving? Paper presented at the mathematics education into the 21 st century project: Proceedings of the international 
conference: the decidable and the undecidable in mathematics education, Brno, Czech Republic, September 2003.

Singer, F. M., \& Voica, C. (2008). Between perception and intuition: learning about infinity. Journal of Mathematical Behavior, 27, 188-205.

Tall, D. O. (2001). Natural and formal infinities. Educational Studies in Mathematics, 48, 199-238.

Tall, D. O. (2007). Developing a theory of mathematical growth. Zentralblatt für Didaktik der Mathematik (ZDM Mathematics Education), 39, 145-154.

Tall, D. O., \& Tirosh, D. (2001). Infinity-the never-ending struggle. Educational Studies in Mathematics, 48(2\&3), 199-238.

Yopp, D. A., Burroughs, E. A., \& Lindaman, B. J. (2011). Why it is important for in-service elementary mathematics teachers to understand the equality $.999 \ldots=1$. Journal of Mathematical Behavior, 30, 304-318. 